\title{
Conical Structures on Acrylic Intraocular Lens (IOLs) Materials after 193-nm Excimer Laser Ablation
}

\author{
Ellas Spyratou $^{{ }^{*}}$, Mersini Makropoulou ${ }^{1}$, Dionisia Tsoutsi ${ }^{2}$, George Zoulinakis ${ }^{1}$, \\ Constantinos Bacharis ${ }^{1}$, Ioannis Asproudis ${ }^{3}$, Alexander Serafetinides ${ }^{1}$ \\ ${ }^{1}$ Department of Physics, School of Applied Mathematical and Physical Sciences, Zografou Campus, National Technical University of \\ Athens, Athens, Greece; ${ }^{2}$ Department of Chemistry, University of Ioannina, Ioannina, Greece; ${ }^{3}$ Department of Ophthalmology, Uni- \\ versity Hospital of Ioannina, Ioannina, Greece. \\ Email: *ellas5@central.ntua.gr
}

Received February $9^{\text {th }}, 2012$; revised March $11^{\text {th }}, 2012$; accepted April $15^{\text {th }}, 2012$

\begin{abstract}
Intraocular lens (IOLs) implants are synthetic lenses used to replace the natural lens of the eye and obtain optical rehabilitation. The materials and methods of IOLs fabrication have been correlated with postoperative complications such as diffractive aberrations, capsular opacification or discoloration. Thus, several new materials and patterns are studied for the formation and etching of intraocular lenses (IOLs). In our work, we studied the use of UV laser as an alternative method to conventional surface shaping techniques for IOLs etching. Ablation experiments were conducted on hydrophobic acrylic IOLs by a commercial excimer laser system used in photorefractive surgery. The morphology of the irradiated area was observed by scanning electron microscopy (SEM) and a mathematical algorithm was used for SEM image processing. The effect of IOLs exposure to UV light before excimer laser irradiation was also examined, since natural ageing and cross-linking of IOLs material were reported. Conical structures were revealed after UV laser ablation and their population was increased with the number of laser pulses. Period distribution of cones was measured with the combination of image processing and a scanning algorithm which was developed for this reason. According to the graphs, the mean period and the distribution of the cones was depending of the number of irradiation pulses and the exposure to UV lamp before laser irradiation. Although a photochemical and a theoretically smooth-surface ablation mechanism is considered for the UV excimer laser interaction with polymers, surface conical-like abnormalities and thermal degradation of the lenses materials was observed.
\end{abstract}

Keywords: Excimer Laser Ablation; UV Light Irradiation; Intraocular Lenses; SEM; Conical Structures

\section{Introduction}

Human prosthetic lens replacements are the most efficient technique to restore vision after cataract surgery or in cases of high levels myopia or hyperopia. Intraocular lenses (IOL) are implanted in the eye to replace the natural crystalline lens through small incisions. However, accommodation is lost with an IOL implant and the patients have had to choose whether they want to have their distance vision corrected by the IOL and wear reading glasses to see up close or to select a power that would permit them to be near-sighted through the IOL and wear a distance correction in their spectacles. A few patients also choose a monovision strategy in which one eye is implanted with an IOL for distance vision and the other with an IOL power that can provide some near vision without spectacle correction $[1,2]$. To provide the ability to see at different distances again, multifocal IOLs have

${ }^{*}$ Corresponding author. been developed $[3,4]$.

Several materials and patterns are still studied for the formation and etching of IOLs in order to improve the quality of vision postoperatively [1,5]. Ophthalmology is entering a very interesting period with new diffractive multifocals, improved refractive multifocals, and accommodative lenses, all coming out at the same time. There are many studies in progress and future will prove the better option. A new diffractive-refractive design for providing intermediated vision is apodization. In an apodized pattern, physical diffractive step heights reduce in height in an almost continuously varying manner from $1.3 \mu \mathrm{m}$ high centrally to $0.2 \mu \mathrm{m}$ high peripherally $[1,6]$. These lenses are either made of PMMA, dyed with a ultra-violet blocking substance, or of newer more flexible materials such as silicone and acrylic which can be folded and placed through a smaller incision in the eye [7-9].

Our study was based on the idea of using laser ablation to modify the surface of intraocular lenses, as it seems 
very intriguing to apply laser radiation for the creation of micron-sized apodized patterns in soft polymers. This could provide an alternative route for IOL's material cutting and etching, instead of using the conventional techniques of injection molding and lathe-cut, which have been correlated with some postoperative complications such as posterior capsular opacification [1,10,11].

The ArF excimer laser remains the dominant source for ablating cornea in refractive surgery, as well as for reshaping polymers, while the UV harmonics of conventional solid-state lasers are also investigated as alternative sources [12,13]. Excimer laser ablation has been reported as an efficient microfabrication technique suitable for surface structuring of polymers because of their high UV absorption $[12,14]$. This technique compete other conventional fabrication methods like embossing, injection molding, lithography and e-beam writing.

As a consequence of modern IOL applications, new photorefractive procedures include the controllable reshaping of organic polymers as an alternative to corneal tissue reshaping $[15,16]$. Hydrophobic acrylic intraocular lenses were ablated by an excimer laser system used in photorefractive surgery for cornea surface modification. Intraocular lenses are polymeric materials which can undergo crosslinking by UV light irradiation. Photocrosslinking and then laser ablation or vice versa, has been studied for the fabrication of arrays microstructures on novel photopolymers [17].

In our previous work, a UV solid state laser has been used for IOL's ablation. The $4^{\text {th }}$ harmonic of an Nd:YAG laser $(\lambda=266 \mathrm{~nm})$ has been examined as a tool for IOL resurfacing and has revealed the contribution of a photothermal and photochemical ablation mechanism [18]. In this work, excimer laser interaction with IOLs surface has been studied. Furthermore, we examined the combination of the two processes, laser ablation and photocrosslinking, in IOLs material modification.

\section{Materials and Methods}

Laser ablation experiments were performed on comercially available hydrophobic acrylic IOLs (Alcon MA30AC, MA30BA and MA60BM models) and yellow azodye IOLs (Alcon SN60WF and SN60AT). The yellow intraocular lenses are designed to reduce the absorption of UV and short wavelength (400 - $500 \mathrm{~nm}$ ) visible light by adding a yellow dye to the IOL and a UV blocking material $[19,20]$. The laser source was a commercial excimer laser (ESIRIS, SCHWIND) providing laser pulses at $\lambda=193 \mathrm{~nm}$ wavelength with pulse duration $8 \mathrm{~ns}$ (Figure 1). The IOLs were placed perpendicular to the incident laser beam and the laser fluence was calculated at $0.5 \mathrm{~J} / \mathrm{cm}^{2}$. Comparative ablation experiments were performed on IOLs which were exposure to UV light before laser irradiation. The possible change in UV laser modification of IOLs surface by their exposure to UV light was investigated. The intraocular lenses have been irradiated with a UV lamp (IROC) emitted at $370 \mathrm{~nm}$ for 15 - $30 \mathrm{~min}$ with intensity $2.20 \mathrm{~mW} / \mathrm{cm}^{2}$. The morphology of ablated IOLs surfaces were examined in detail with the use of a scanning electron microscope (SEM, JEOL JSM 5600).

\section{Results and Discussion}

Ablation experiments on hydrophobic acrylic and yellow azo-dyed intraocular lenses were conducted with the excimer laser $\left(\lambda=193 \mathrm{~nm}, \mathrm{t}_{\mathrm{p}}=8 \mathrm{~ns}\right)$ at low laser fluence $0.5 \mathrm{~J} / \mathrm{cm}^{2}$ and various number of pulses. In the UV and

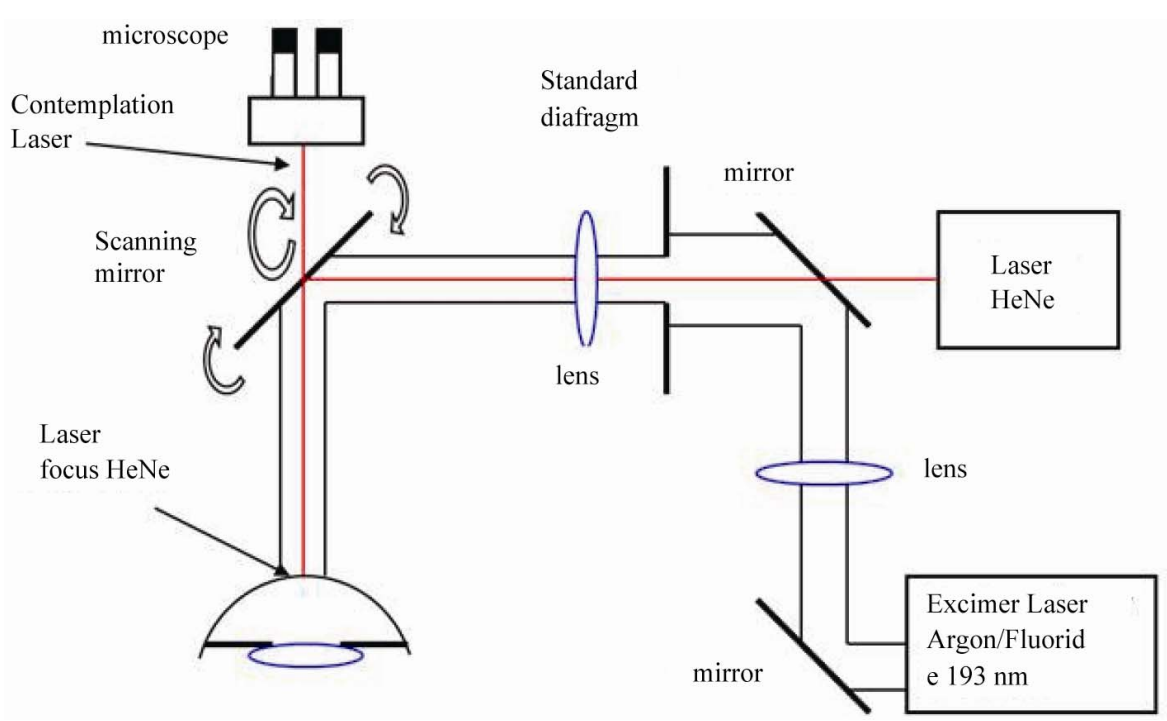

(a)

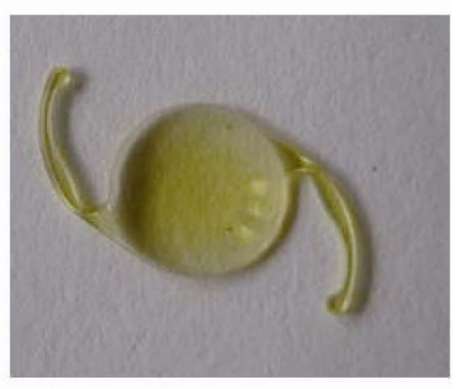

(b)

Figure 1. (a) Schematic presentation of the excimer laser system (ESIRIS); (b) Image of a yellow intraocular lens. 
VUV, the ablation threshold of polymers can be very low, with typical values ranging from $\mathrm{F}_{\text {th }} \sim 20-200 \mathrm{~mJ} / \mathrm{cm}^{2}$, depending on the laser wavelength as well as the polymer itself $[21,22]$. Irradiation was performed at normal incidence angle. The morphology of the ablated hydrophobic acrylic and yellow azo-dyed intraocular lenses was investigated with SEM. Conical structures were revealed after irradiation and their population was increased with the number of pulses. In Figure 2(a) is presented the SEM image of an acrylic IOL (Alcon MA60BM model, $21.0 \mathrm{D})$ after irradiation with excimer laser at laser fluence $0.5 \mathrm{~J} / \mathrm{cm}^{2}$ and 3000 pulses. A few conical structures appeared around the crater walls with cracks on them. A larger magnification of one of the cones is shown in

\section{Figure 2(b).}

Figures 3(a)-(d) show the SEM images of an acrylic IOL (Alcon MA30AC model, 21.5 D) after irradiation with excimer laser at laser fluence $0.5 \mathrm{~J} / \mathrm{cm}^{2}$ and 7650 pulses. In Figures 3(a) and (b) are presented the plane view of the IOL ablation crater at different magnifications. Cracks were observed at the edge of the crater and might be due to high pressure that exceeds the mechanical strength of the material. The black hole in the bottom of the crater is the perforated area of the IOL. For normal incidence observation, spherical-like structures appeared inside and outside the crater. However, under tilted angle observation, as is shown in Figure 3(c), a conical structure formation was revealed. The cones appear around
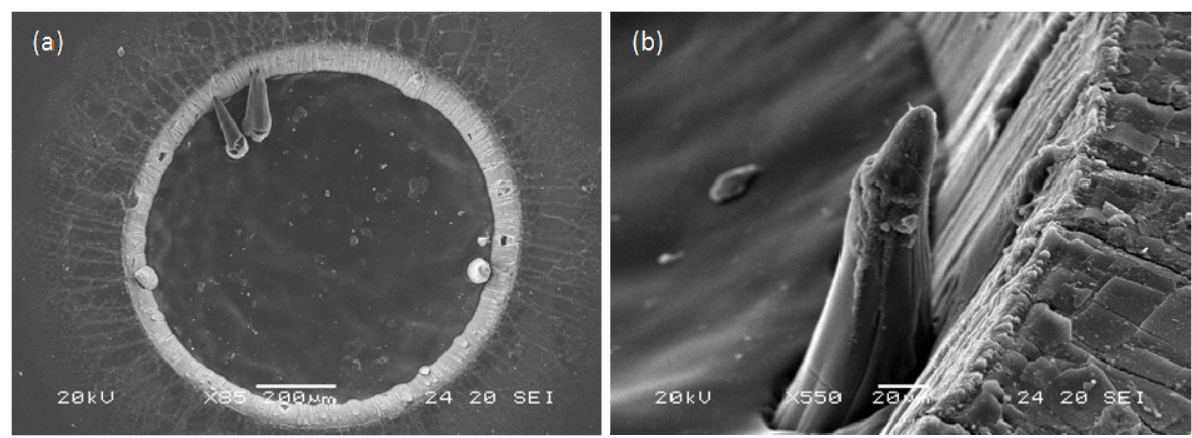

Figure 2. SEM images of IOL (Alcon MA60BM model, $21.0 \mathrm{D}$ ) after irradiation with excimer laser at laser fluence $0.5 \mathrm{~J} / \mathrm{cm}^{2}$ and 3000 pulses. (a) A plane section of the ablated crater (under $\times 85$ magnification); (b) Detailed image of a conical structure near the walls of the ablated crater.
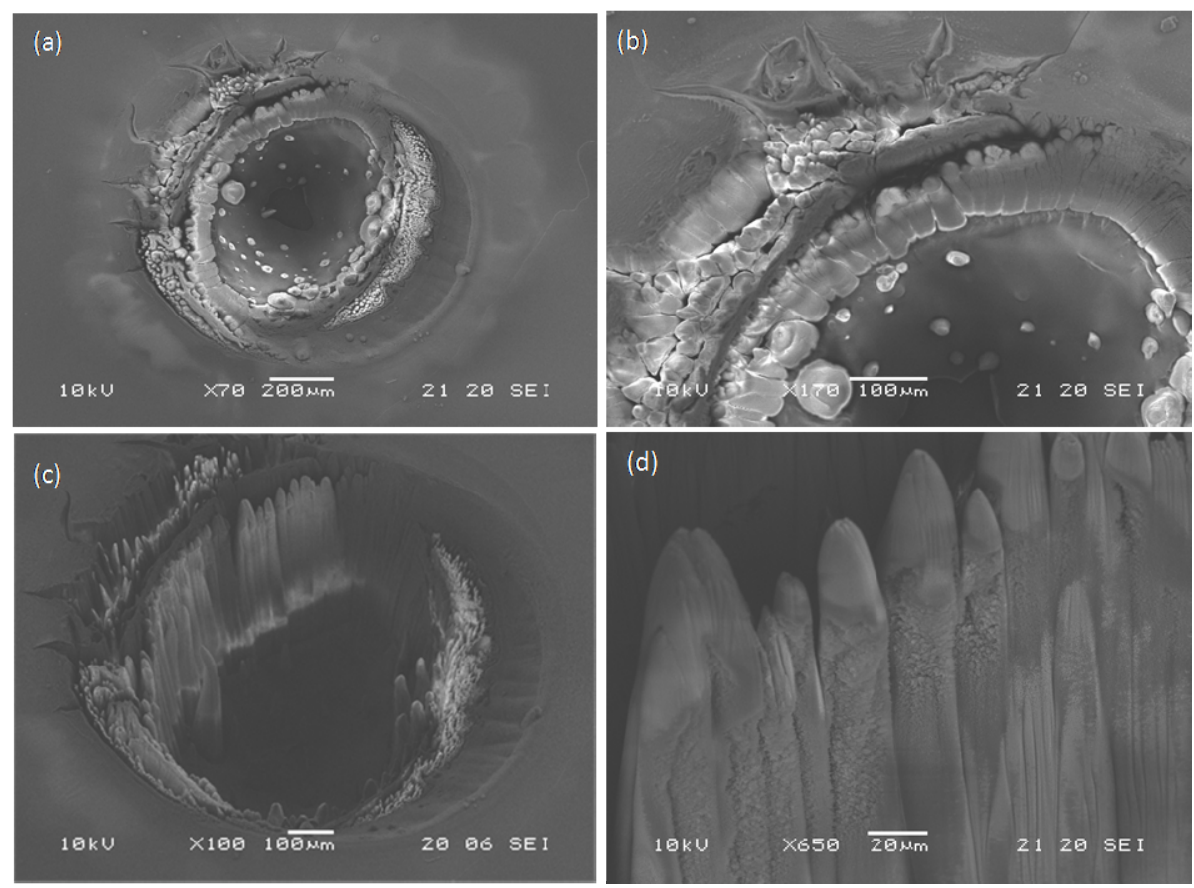

Figure 3. SEM images of IOL (Alcon MA30AC model, $21.5 \mathrm{D}$ ) after irradiation with excimer laser at laser fluence $0.5 \mathrm{~J} / \mathrm{cm}^{2}$ and 7650 pulses. (a) A plane section of the ablated crater (under $\times 70$ magnification); (b) Detailed image of crater edges (under $\times 170$ magnification) for normal incidence observation, (c) A tilted angle observation of the crater shows a conical structure formation; (d) Higher magnification of the cross-section of the cones $(\times 650)$. 
the walls and oriented vertical to the bottom of crater. In Figure 3(d) is observed the cone-like microstructure under higher magnification. The cones present smooth edges and rougher lateral surface.

Figures 4(a) and (b) present the SEM images of an acrylic IOL (Alcon MA60BM model, 21.0 D) after irradiation with excimer laser at laser fluence $0.5 \mathrm{~J} / \mathrm{cm}^{2}$ and 11,000 pulses. An evolution of the surface conical microstructure growth was observed with the increased number of pulses. The feature of the cones is a smooth edge with sharp and intense apex (Figure 4(c)). However, higher magnification of the cone edges reveals nonspherical nanostructures around the cone vertices and spherical nanoparticles on the top of the cone. The nonspherical debris can be associated with polymer and carbon fragments and the spherical one with pure carbon fragments during the laser ablation [23].

In an effort to explain these interesting patterns, observed for the best of our knowledge for the first time in intraocular lens polymeric samples, at low UV laser energy fluence and high number of laser pulses, we reviewed some relatively similar results in other polymer applications from literature. Several works on excimer laser irradiation of polymeric materials reported on the formation of conical shape structures that are formed for a selected range of laser parameters (fluence and number of pulses), especially in the 1990 decade, regarding microelectronics and photolithography applications [12,21-
$25,29]$. For example, similar conical structures were also observed during ablation of poly(2-hydroxyethyl methacrylate) (PHEMA) by an ArF excimer laser $(\lambda=193 \mathrm{~nm})$, with 8000 pulses at $180 \mathrm{~mJ} / \mathrm{cm}^{2}$ [24]. Excimer laser ablation of elastomer composite containing carbon black produced cone-like microstructures on the surface after irradiation with a $\mathrm{KrF}$ excimer laser $(\lambda=248 \mathrm{~nm}, 30 \mathrm{~ns})$ at $0.5 \mathrm{~J} / \mathrm{cm}^{2}$ and for $100-500$ pulses [25]. Interaction of intense ultrashort laser pulses (120 fs at $\lambda=795 \mathrm{~nm}$ ) with polymer based composites has revealed cone-like structures on the whole walls at $5.4 \mathrm{~J} / \mathrm{cm}^{2}$ and for $50-90$ pulses [26]. Sparsely dispersed cones were observed after extreme ultraviolet (EUV) radiation for $60 \mathrm{~s}$ with $10 \mathrm{~Hz}$ repetition rate at $10 \mathrm{~mJ} / \mathrm{cm}^{2}$ [27]. Recently, formation of a large number of cones was also observed after $248 \mathrm{~nm}$ excimer laser irradiation on samples of wooden archaeological objects with laser fluence ranging from 40 up to $1300 \mathrm{~mJ} / \mathrm{cm}^{2}$ and with a number of pulses in the range from 10 to 10,000 . For the lowest value of $40 \mathrm{~mJ} / \mathrm{cm}^{2}$, and thousands of pulses, SEM observations in consolidant acrylic resins (Plexisol 550 and Paraloid B72) revealed morphological changes with formation of a large number of cones, restricted to the very few surface layers [28], while, at the highest value of $1300 \mathrm{~mJ} / \mathrm{cm}^{2}$ and with a few laser pulses (10), only wood ablation without surface morphological change was observed.

Hopp [29] proved that the development of conical structures is due to the shadowing and diffraction effect,
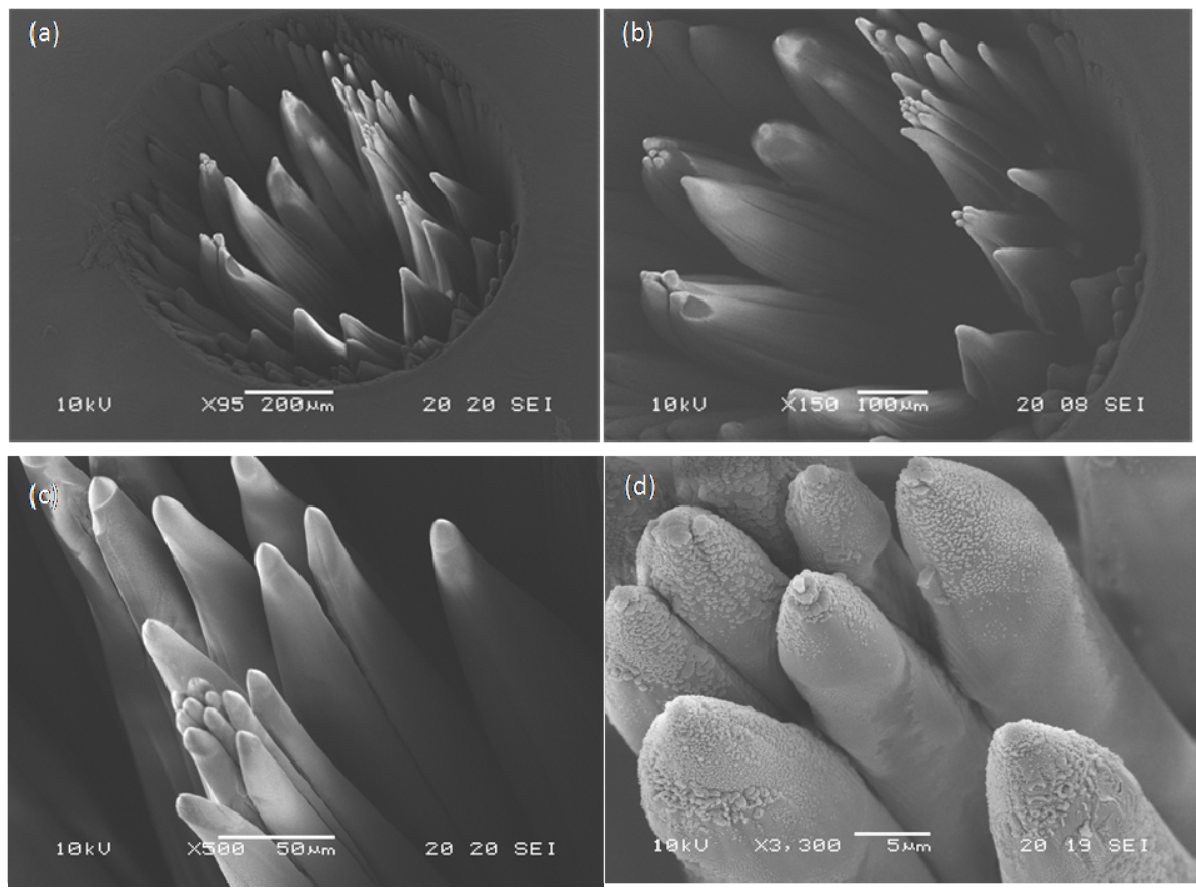

Figure 4. SEM images of acrylic IOL (Alcon MA60BM model, 21.0 D) after irradiation with excimer laser at laser fluence 0.5 $\mathrm{J} / \mathrm{cm}^{2}$ and $\mathrm{N}=11,000$ pulses. (a) and (b) Conical microstructures with dense dispersion appeared over the ablated crater; (c) A tilted angle observation of the cones presenting smooth edge and intense apex; (d) Higher magnification of the cone vertices reveals non-spherical nanostructures around the cone vertices and spherical nanoparticles on the top of the cone. 
induced by the particulate impurities, and the interference effect between the incident and reflecting (from particulate impurities being on the ablated surface) parts of the UV laser beam, in a well established range of the laser parameter values [29]. The origin of the impurities could be attributed to imperfections which pre-existed in the material or created during laser ablation. UV photons of $6.4 \mathrm{eV}(\lambda=193 \mathrm{~nm})$ have sufficient energy to induce main polymer chain scission and side chain scission. Photon cleavage of the main chain bond leads to decomposition of the polymer into chains with lower molecular weight or even monomers. Photon cleavage at the ester carbonyl group results in the formation of carbon radicals on the main chain, thus leading to cross-linking of polymers as well as main chain scission and monomer elimination [30]. The carbon particles formed inside the laser plume, can settle on the composite surface provide a shielding effect having a higher threshold than the polymer. Thus, conical morphologies are created with repeated pulses. It is important to note that on the peak of the cones often particulate impurities can be seen (Figure 3(d)). Likewise, interference fringes have been observed at the base of the cone. The fringes are believed to be the result of the interference between the incident beam and the one reflected from the surface of the cone [29].

In Figure 5 are presented the SEM images of an IOL (MA30BA model, 21.5D) which was exposed in UV light before the effect of laser ablation. The IOL has been irradiated with a lamp at $\lambda=370 \mathrm{~nm}$ for $30 \mathrm{~min}$, with an intensity of $2.20 \mathrm{~mW} / \mathrm{cm}^{2}$. The ablation experiment was conducted by excimer laser at $0.5 \mathrm{~J} / \mathrm{cm}^{2}$ and 7190 pulses. The plane and the cross section of the crater are shown in Figures 5(a) and (b) respectively. The perforation of the lens was realized with 7190 pulses and was observed near the centre of the crater's bottom. No conical microstructure appeared inside the crater but only few cones near the crater walls and more embedded with crater walls. Some debris noticed inside the crater was attributed to dust and not to ejected ablated material. Figure 5(c) shows in detail the growth of cones indicated by the framed area of Figure 5(a). The evolution of shape microstructures which tend to create cones is shown in high magnification in Figure 5(d)). The conical-like structures are embedded in crater walls and appear a rough surface with intense ripples.

In Figures 6 and 7, we observed SEM images of the laser ablation effect on "yellow azo-dye" IOLs without pre-exposure to UV lamp and after pre-exposure to UV irradiation, respectively. Conical structures were also appeared in both cases. A dense dispersion of cones appeared for $\mathrm{N}=11,000$ pulses (Figure 6(a)) and sparsely dispersed cones for less pulses $\mathrm{N}=6500$. The IOL in Figure 6 was exposed in UV lamp for 15 min with an intensity $2.20 \mathrm{~mW} / \mathrm{cm}^{2}$. Cones have perpendicular orientation as the beam falls vertical to the IOL surface. However, some conical structures present a slight tilt (Figure 6(b)) which is attributed to the beam aberration
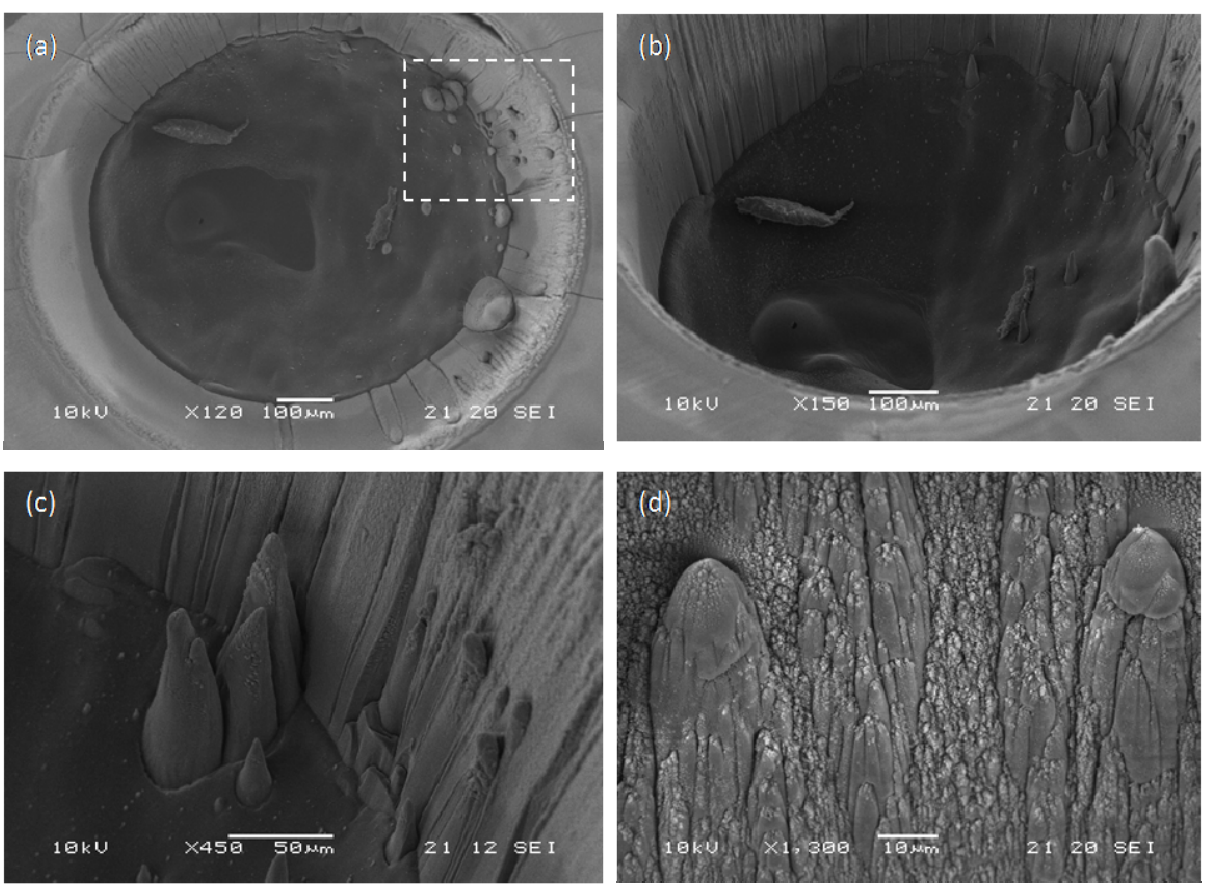

Figure 5. A SEM image of acrylic IOL (Alcon MA30BA model, 21.5 D) after was exposed in UV light before laser irradiation. (a)-(b) Plane and cross section of the ablated crater with excimer laser at laser fluence $0.5 \mathrm{~J} / \mathrm{cm}^{2}$ and $\mathrm{N}=7190$ pulses, respectively; (c) Detail of the cones growth indicated by the framed area of (a); Conical-like structures embedded in crater walls (d). 

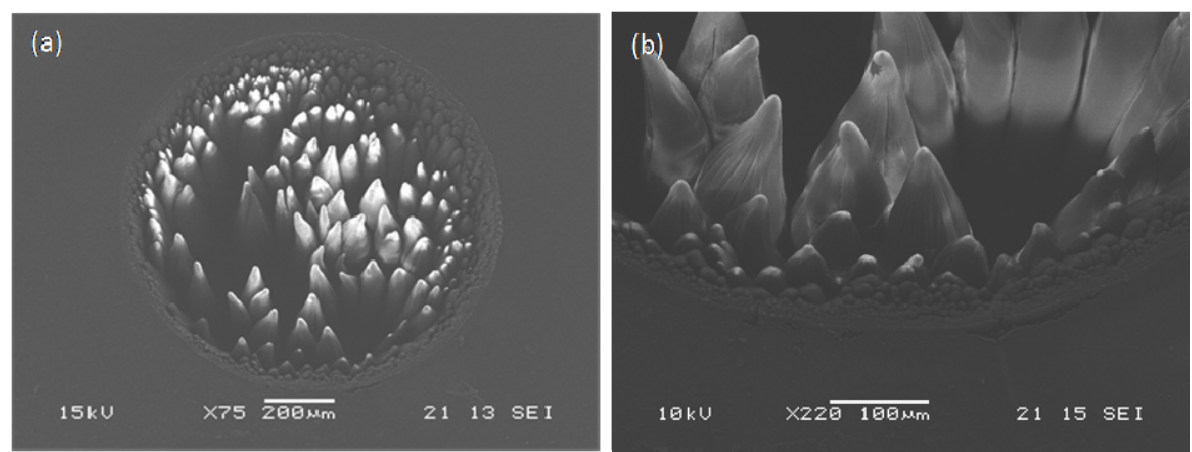

Figure 6. SEM images of a yellow azo-dye acrylic IOL (Alcon SN60WF model, 21.5 D) after irradiation with excimer laser at laser fluence $0.5 \mathrm{~J} / \mathrm{cm}^{2}$ and $\mathrm{N}=11,000$ pulses. (a) Conical microstructures with dense dispersion appeared over the ablated crater; (b) Some cones show a slight tilt due to IOL's curvature.
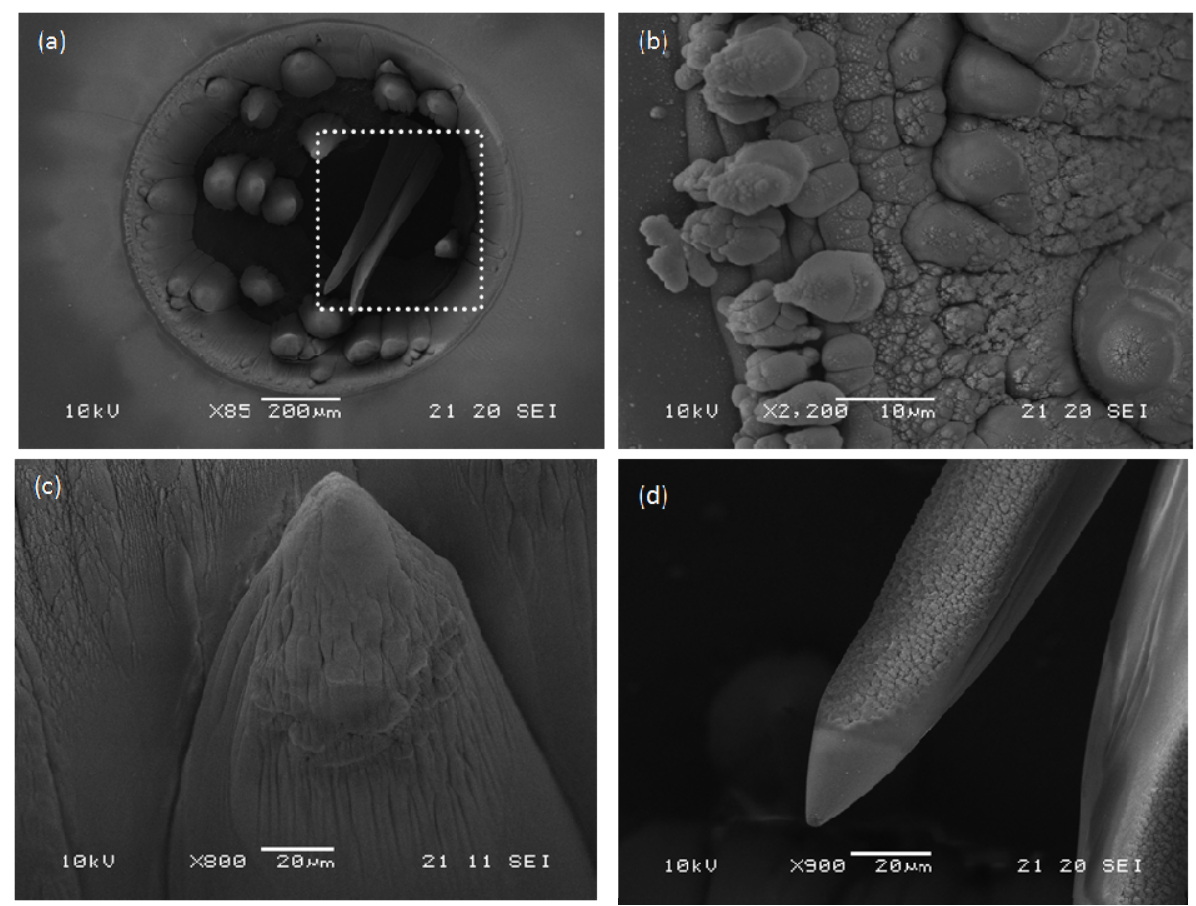

Figure 7. SEM images of a yellow azo-dye acrylic IOL (Alcon SN60AT model, 21.0 D) pre-exposed in UV light before excimer laser irradiation at laser fluence $0.5 \mathrm{~J} / \mathrm{cm}^{2}$ and $\mathrm{N}=6500$ pulses. (a) An intense tilt of two cones which tend to collapse inside the crater hole as indicated by the framed area; (b) Nascent conical structures appeared around the walls; (c)-(d) The cones appear relative smooth apex and rough lateral surface.

as the IOL is not flat but has a curvature determined by the diopters. The framed area of Figure 7(a) shows the intense tilt of two cones which tend to collapse inside the crater hole. Conical structure formation was noticed around the crater walls. Nascent conical structures are shown around the walls in Figure 7(b). The cones present relative smooth apex, while nanostructures were created on the lateral surface associated with polymer and carbon fragments during the material ablation (Figures $7(\mathbf{c})$ and (d)). Similar behavior was observed for IOL's surface irradiation at the same laser fluence but with few pulses $(50-100)$. A budding of conical-like structures was observed under atomic force microscopy analysis (unpublished data).

In all case, plasma formation and thermal damage zone around the ablation craters was observed during the irradiation. The thermal alteration was visible even by naked eye or conventional light microscopy. Although, the UV excimer laser ablation mechanism is generally considered as a "cold" mechanism, the morphological appearance of the IOL samples reveals evidence of a thermal degradation of IOLs and, therefore, the contribution of a photothermal mechanism in IOLs ablation. It was reported $[21,31,32]$ that both photochemical and photothermal mechanism contribute in the UV organic polymer ablation at high absorption wavelengths of $193 \mathrm{~nm}$ and 
$248 \mathrm{~nm}$. The electronic energy is converted to heat via radiationless transitions followed by thermal "unzipping" of the polymer [33].

In our previous report on UV laser ablation (at $\lambda=266$ $\mathrm{nm}$ ) of IOL [18], we indicated that neither an exclusively photochemical or thermal ablation process is appropriate in UV laser polymer ablation and that both factors may be potentially important. Ultraviolet photons with energies higher than $3.5 \mathrm{eV}$ give rise to electronic excitation, which can lead to photochemical decomposition of polymers [34]. So, incident photons of $266 \mathrm{~nm}$ have sufficient high energy $(4.7 \mathrm{eV})$ to directly break main chain bonds. Bond-breaking and material ejection is believed to occur directly from the excited electronic state in the photochemical mechanism $[33,35]$.

Period distribution of cones was measured with the combination of image processing and a scanning algorithm which was developed for this reason. The image processing of SEM images was produced to relate each pixel with the apex of each cone. Morphological filters were used for image processing. In Figure 8 is shown a characteristic example of the image processing of the SEM image of the cones in Figure 6.

According to the graphs of Figures 9-14, the mean period and the distribution of the cones is reducing as the number of irradiation pulses of acrylic IOLs is increasing. For the same number of pulses, the IOLs which have been experienced "artificial ageing" presented wider distribution I comparison with IOLs which have not been exposure to UV lamp before excimer laser irradiation.

The algorithm can produced the mean value of period and distribution of the conical structures [36]. Thus, it is possible to measure the distance of each cone form the nearest one or in other words the distance between two nearest pixels. The results of the algorithm were extracted with the form of a graph.

In Figure 15 we present a schematic representation for the creation of cones, inspired by the work of Silvain et al. [23] for the cone-like microstructures induced by $\mathrm{KrF}$

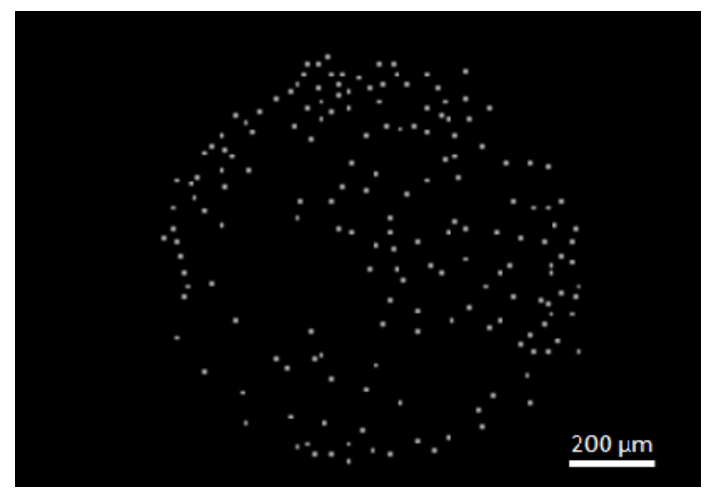

Figure 8. Image processing with morphological filters of the SEM image of Figure 5(a).

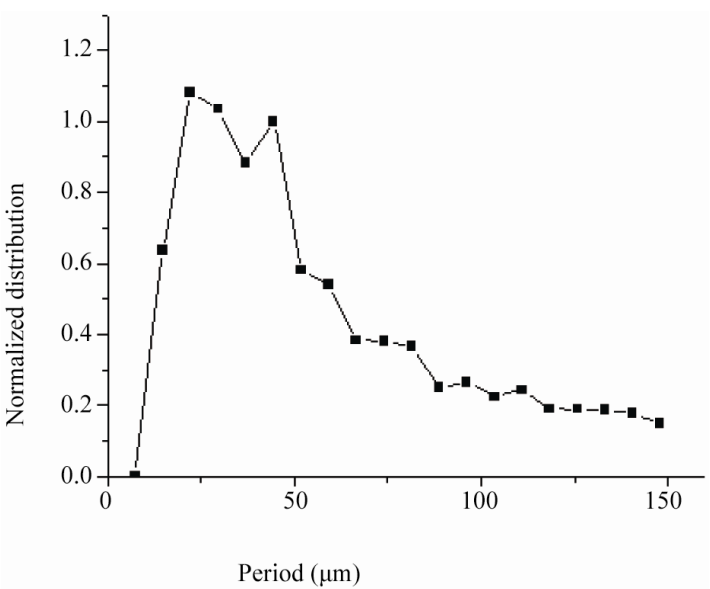

Figure 9. Graph of cones distribution correspond to the irradiation of the yellow SN60WF IOL with $\mathrm{N}=11.000$ pulses and pre-exposure to UV lamp.

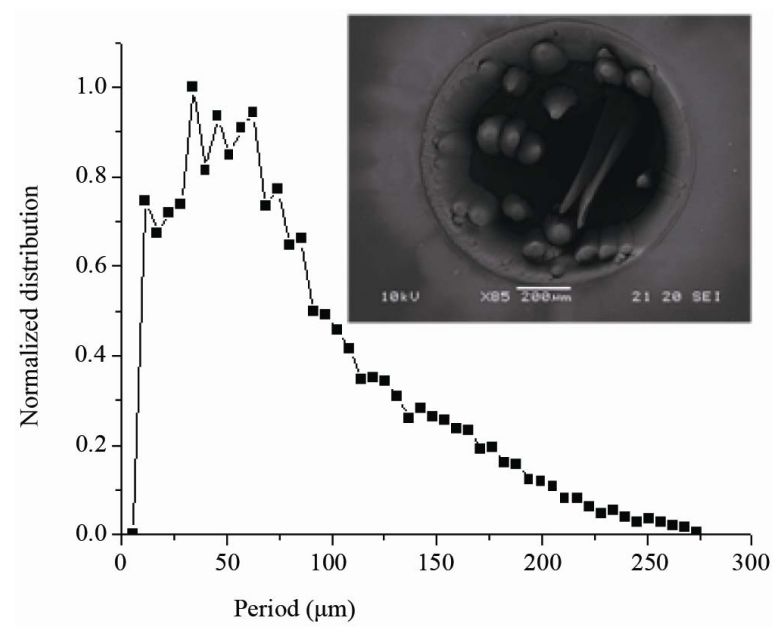

Figure 10. Graph of cones distribution correspond to the irradiation of the acrylic MA30BA IOL with $N=7.189$ pulses and pre-exposure to UV lamp.

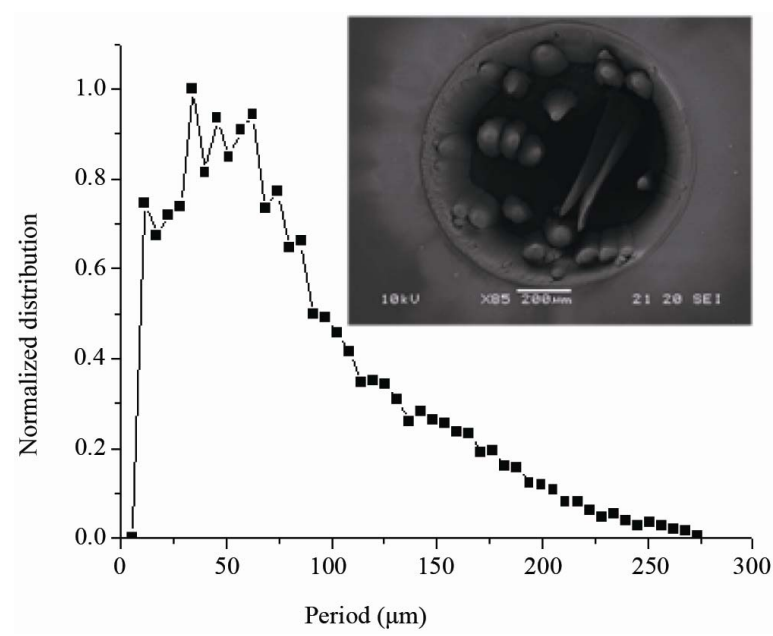

Figure 11. Graph of cones distribution correspond to the irradiation of the yellow SN60WF IOL with $N=6.500$ pulses and pre-exposure to UV lamp. 


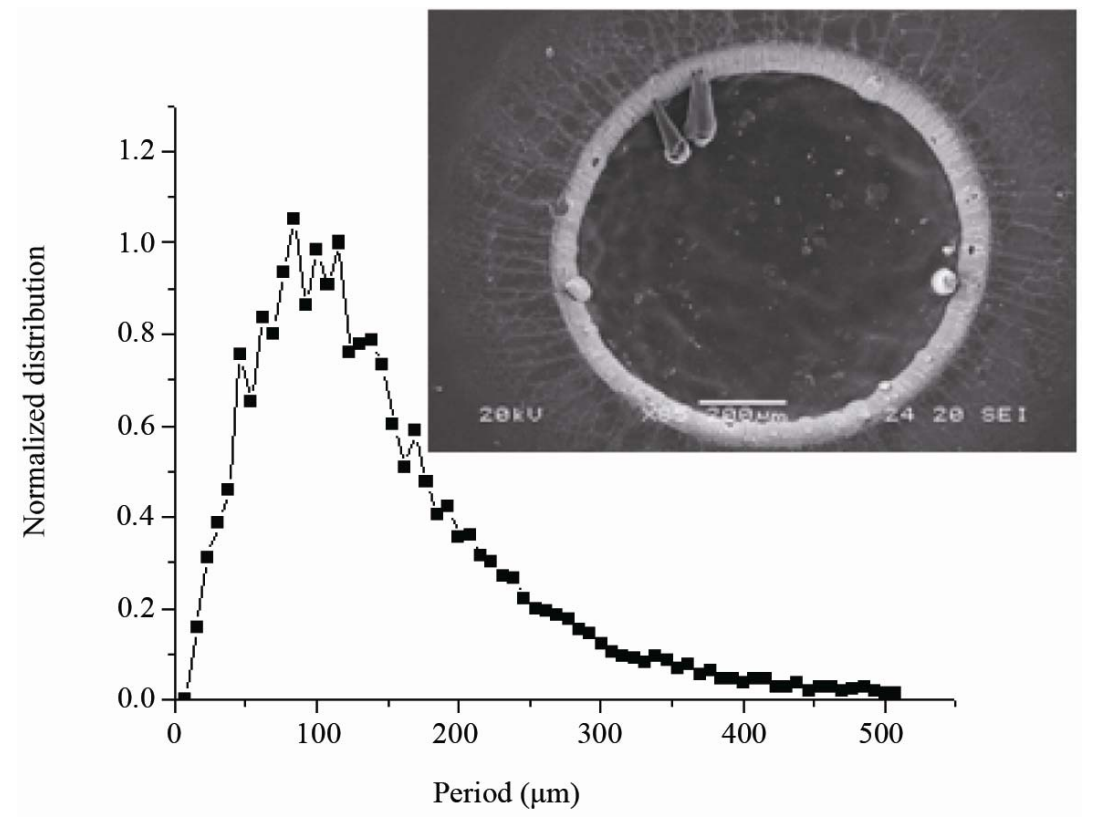

Figure 12. Graph of cones distribution correspond to the irradiation of the acrylic MA60BM IOL with $N=3.000$ pulses and without exposure to UV lamp.

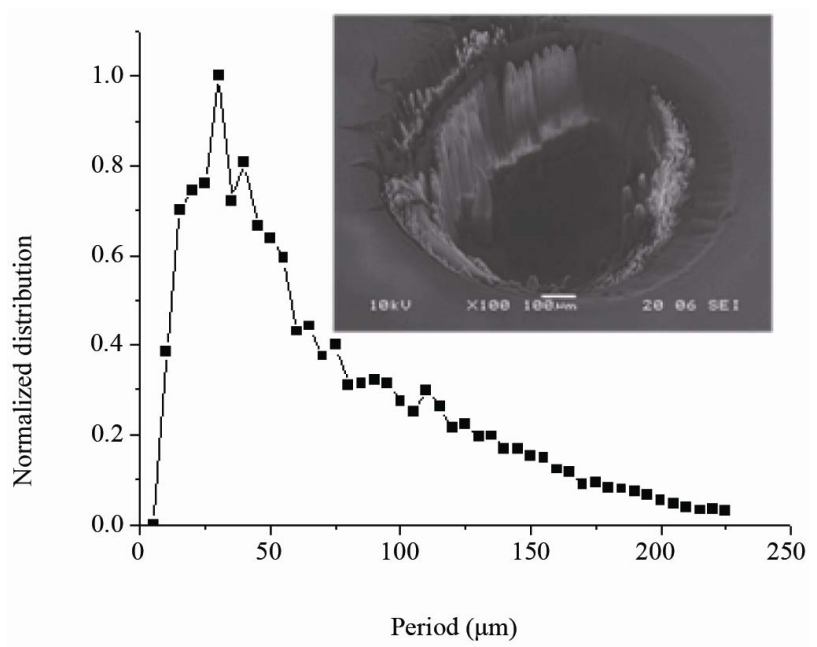

Figure 13. Graph of cones distribution correspond to the irradiation of the acrylic MA30AC IOL with $\mathrm{N}=7.650$ pulses and without exposure to UV lamp.

excimer laser irradiation of composite materials. The molten layer of the material can induce motion of the surface micrometer carbon particles until its solidification. For the first ablation pulses carbon particles are free to move randomly on the ablated surface and therefore to make clusters. Each carbon particles aggregation can act as shield for the growth of cone-like microstructure. Growth mechanism of the large cone-like structure can then take place through the aggregation of small conelike microstructures in thick molten layer upon repeated laser irradiation. The exposure of IOL to UV light induces cross-linking between polymer chains. The resulting modification of mechanical properties depends strongly on the cross-link density. Low cross-link densities, for $2.20 \mathrm{~mW} / \mathrm{cm}^{2}$ UV lamp irradiation, raise the viscosity of melting polymer. The limited diffusion of carbon particles through the strong-net polymer chains and inside the molten layer can lead to formation of bulk cones.

\section{Conclusions}

Intraocular lenses modification or etching was examined by the use of excimer laser irradiation at $193 \mathrm{~nm}$. The ablated surfaces present conical microstructures attributed to carbon particles impurities which act as shielding effects. Although the photochemical ablation mechanism of the UV laser thermal degradation of the lenses materials was observed. The acrylic material of the lenses exhibit nearly the same morphology after crosslinking them by exposure to UV lamp before UV laser irradiation. The formation of cone-like structure during ArF irradiation is associated with the diffusion and aggregation of carbon particles. Growth mechanism of large cone-like structure can then follow to aggregate small cone-like microstructures in thick molten layer upon repeated laser irradiation.

For IOL surface modification, a properly smooth surface ablation patterning is absolutely necessary. Therefore, a systematic study and careful control of laser parameter radiation in combination with IOLs properties may eliminate the rough conical structures before inserting UV laser irradiation as a useful micromachining for "designer" IOLs. 


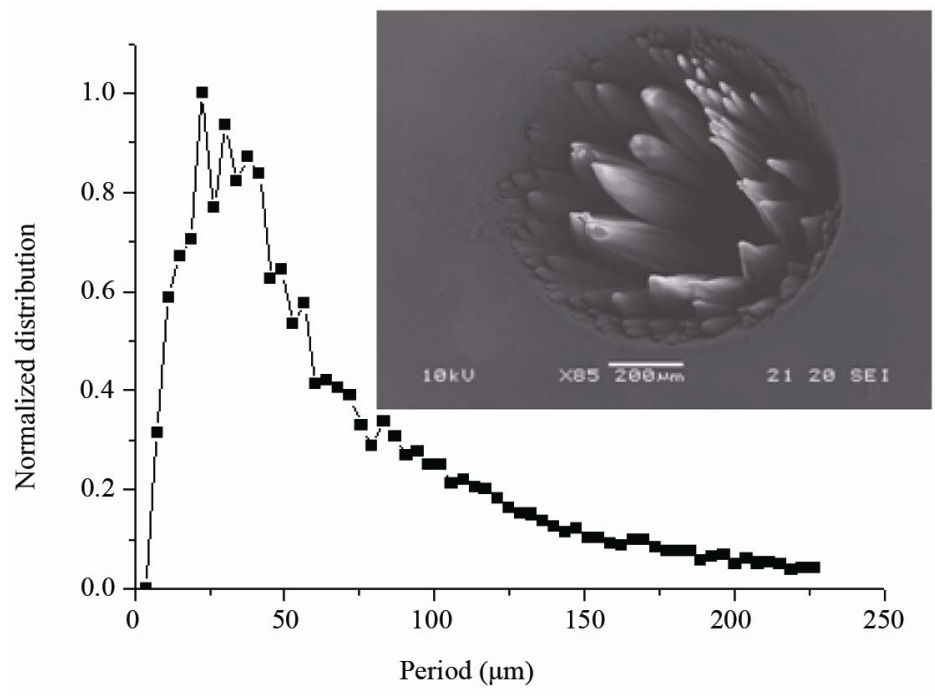

Figure 14. Graph of cones distribution correspond to the irradiation of the acrylic MA60BM IOL with $N=11.000$ pulses and without exposure to UV lamp.

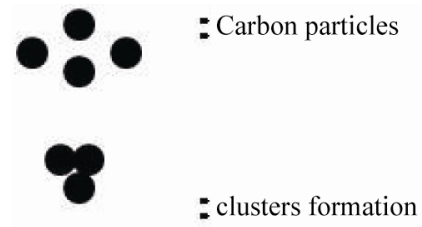

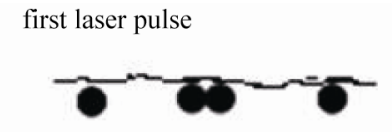

second pulse
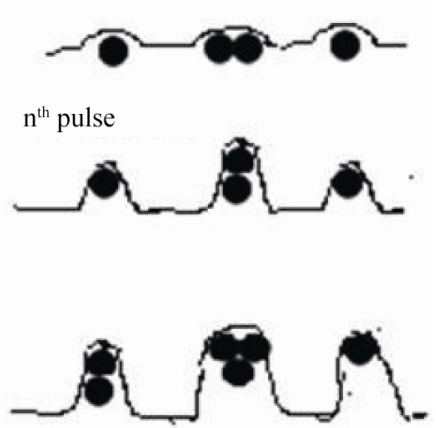

Figure 15. Schematic image of the small and large conical microstructures growth under the effect of excimer laser irradiation on IOLs.

\section{Acknowledgements}

The authors would like to thank Prof. F. Pomonis for his help with the SEM examination. Thanks are due to Mr. Th. Alexopoulos, from System Vision Inc., for providing us the access to the ESIRIS excimer laser. Thanks are also due to Dr. Sp. Georgaras for providing us intraocular lenses and to Dr. V. Tsigos for the access to UV irradiation facility.

\section{REFERENCES}

[1] J. A. Davison and M. J. Simpson "History and Development of the Apodized Diffractive Intraocular Lens," Journal of Cataract and Refractive Surgery, Vol. 32, No. 5, 2006, pp. 849-858. doi:10.1016/j.jcrs.2006.02.006

[2] S. Jain, I. Aurora and D. T. Asar, "Success of Monovision in Presbyopes: Review of Literature and Potential Applications to Refractive Surgery," Survey of Ophthalmology, Vol. 40, No. 6, 1996, pp. 491-499. doi:10.1016/S0039-6257(96)82015-7

[3] J. C. Javitt and R. F. Steinert, "Cataract Extraction with Multifocal Intraocular Lens Implantation: A Multinational Clinical Trial Evaluating Clinical, Functional, and Quality-of-Life Outcomes," Ophthalmology, Vol. 107, No. 11, 2000, pp. 2040-2048. doi:10.1016/S0161-6420(00)00368-7

[4] H. N. Sen, A. U. Sarikkola, R. J. Uusitalo and L. Laatikainen, "Quality of Vision after AMO Array Multifocal Intraocular Lens Implantation," Journal of Cataract and Refractive Surgery, Vol. 30, No. 12, 2004, pp. 2483-2493. doi:10.1016/j.jcrs.2004.04.049

[5] R. J. Olson, L. Werner, N. Mamalis and R. Cionni, "New Intraocular Lens Technology," American Journal of Ophthalmology, Vol. 140, No. 4, 2005, pp. 709-716. 
doi:10.1016/j.ajo.2005.03.061

[6] R. F. Steinert, B. L. Aker and D. J. Trentacost, "A Prospective Comparative Study of the AMO Array ZonalProgressive Multifocal Silicone Intraocular Lens and a Monofocal Intraocular Lens," Ophthalmology, Vol. 106, No. 7, 1999, pp. 1243-1255. doi:10.1016/S0161-6420(99)00704-6

[7] T. Oshika, T. Nagata and Y. Ishii, "Adhesion of Lens Capsule to Intraocular Lenses of Polymethylmethacrylate, Silicone and Acrylic Foldable Materials: An Experimental Study," British Journal of Ophthalmology, Vol. 82, No. 5, 1998, pp. 549-553. doi:10.1136/bjo.82.5.549

[8] V. Sankar, T. K. Kumar and K. R. Punduranga, "Preparation Characterization and Fabrication of Intraocular Lenses from Photon Initiated Polymerized Poly (Methymethacrylate)," Trends in Biomaterial and Artificial Organs, Vol. 17, No. 2, 2004, pp. 24-30.

[9] S. D. McLeod, V. Portney and A. Ting, "A Dual Optic Accommodating Foldable Intraocular Lens," British Journal of Ophthalmology, Vol. 87, No. 9, 2003, pp. 10831085. doi:10.1136/bjo.87.9.1083

[10] M. C. Kraff, D. Sanders and H. L. Lieberman, "Serial Corneal Endothelial Cell Loss with Lathe-Cut and Injection-Molded Posterior Chamber Intraocular Lenses," Journal of American Intraocular Implant Society, Vol. 9, No. 3, 1983, pp. 301-305.

[11] S. E. Wilson and R. F. Brubaker, "Neodymium: YAG Laser Damage Threshold. A Comparison of InjectionMolded and Lathe-Cut Polymethylmethacrylate Intraocular Lenses," Ophthalmology, Vol. 94, No. 1, 1987, pp. 7-11.

[12] P. E. Dyer, "Excimer Laser Polymer Ablation: Twenty Years on," Applied Physics A, Vol. 77, No. 2, 2003, pp. 167-173. doi:10.1007/s00339-003-2137-1

[13] A. A. Serafetinides, M. Makropoulou, E. Fabrikesi, E. Spyratou, C. Bacharis, R. R. Thomson and A. K. Kar, "Ultrashort Laser Ablation of PMMA and Intraocular Lenses," Applied Physics A, Vol. 93, No. 1, 2008, pp. 111-116. doi:10.1007/s00339-008-4666-0

[14] K. Naessens, H. Ottevaere, P. Daele and R. Baets, "Flexible Fabrication of Microlenses in Polymer Layers with Excimer Laser Ablation," Applied Surface Science, Vol. 208-109, 2003, pp. 159-164. doi:10.1016/S0169-4332(02)01359-4

[15] A. Fuxbruner, I. Hemo, A. Lewis, A. Zauberman, D. Blau and D. Polotsky, "Controlled Lens Formation with Unperturbed Excimer Lasers: Use with Organic Polymers and Corneal Tissues," Applied Optics, Vol. 29, No. 36, 1990, pp. 5380-5386. doi:10.1364/AO.29.005380

[16] F. Manns, P. Rol, J. M. Parel, A. Schmid, J. H. Shen, T. Matsui and P. Soderberg, "Optical Profilometry of Poly (Methylmethacrylate) Surfaces after Reshaping with a Scanning Photorefractive Keratectomy (SPRK) System," Applied Optics, Vol. 35, No. 19, 1996, pp. 3338-3391. doi:10.1364/AO.35.003338

[17] T. Lippert, J. Wei, A. Wokaun, N. Hoogen and O. Nuyken, "Development and Structuring of Combined Positive Negative/Negative-Positive Resists Using Laser Ablation as Positive Dry Etching Technique," Macromolecular Materials and Engineering, Vol. 283, No. 1, 2000, pp. 140-
143.

doi:10.1002/1439-2054(20001101)283:1<140::AID-MA ME140>3.0.CO;2-F

[18] E. Spyratou, I. Asproudis, D. Tsoutsi, C. Bacharis, K. Moutsouris, M. Makropoulou and A. A. Serafetinides, "UV Laser Ablation of Intraocular Lenses: SEM and AFM Microscopy Examination of the Biomaterial Surface," Applied Surface Science, Vol. 256, No. 8, 2010, pp. 2539-2545. doi:10.1016/j.apsusc.2009.10.101

[19] Z. X. Yuan, P. Reinach and J. P. Yuan, "Contrast Sensitivity and Colour Vision with a Yellow Intraocular Len," American Journal of Ophthalmology, Vol. 138, No. 1, 1998, pp. 1004-1010. doi:10.1016/j.ajo.2004.02.024

[20] Y. Yanagi, Y. Inoue, A. Iriyama and W. D. Jang, "Effects of Yellow Intraocular Lenses on Light-Induced Upregulation of Vascular Endothelial Growth Factor," Journal of Cataract and Refractive Surgery, Vol. 32, No. 9, 2006, pp. 1540-1544. doi:10.1016/j.jers.2006.04.012

[21] P. E. Dyer, "Laser Ablation of Polymers," In: I. W. Boyd and R. B. Jackman, Eds. Photochemical Processing of Electronic Materials, Academic, London, 1992, pp. 360-385.

[22] H. Sato and S. Nishio, "Polymer Laser Photochemistry, Ablation, Reconstruction, and Polymerization," Journal of Photochemical Photobiology C, Vol. 2, No. 13, 2001, pp. 139-152. doi:10.1016/S1389-5567(01)00015-6

[23] J. F. Silvain, H. Niino, S. Ono, S. Nakaoka and A. Yabe, "Surface Modification of Elastomer Carbon Composite by Nd:YAG Laser and KrF Excimer Laser Ablation," Applied Surface Science, Vol. 141, No. 1-2, 1999, pp. 2534. doi:10.1016/S0169-4332(98)00611-4

[24] T. V. Chirila and P. P Saarloos, "Ablation of Poly (2-Hydroxyethyl Methacrylate) by 193-nm Excimer Laser Radiation," Journal of Applied Polymer Science, Vol. 44, No. 8, 1992, pp. 1355-1363. doi:10.1002/app.1992.070440806

[25] S. Ono, S. Nakaoka, J. Wang, H. Niino and A. Yabe, "Formation of Elastic Cone-Like Microstructures on the Composite of Elastomer and Carbon Black by Excimer Laser Ablation," Journal of Applied Physics, Vol. 36, 1997, pp. 1387-1389. doi:10.1143/JJAP.36.L1387

[26] P. Moreno, C. Méndez, A. García, I. Arias and L. Roso, "Femtosecond Laser Ablation of Carbon Reinforced Polymers," Applied Surface Science, Vol. 252, No. 12, 2006, pp. 4110-4119. doi:10.1016/j.apsusc.2005.06.008

[27] A. Bartnik, H. Fiedorowicz, R. Jarocki, J. Kostecki, A. Szczurek and M. Szczurek, "Ablation and Surface Modifications of PMMA Using a Laser-Plasma EUV Source," Applied Physics B, Vol. 96, No. 4, 2009, pp. 727-730. doi:10.1007/s00340-009-3692-8

[28] R. Belli, L. Toniutti, A. Miotello, P. Mosaner and D. Avi, "Excimer Laser Irradiation at $248 \mathrm{~nm}$ of Wooden Archaeological Objects and Polymeric Consolidants Used in Conservation: A Study of Cone Formation and Optimum Cleaning Parameters," Applied Physics A, Vol. 92, No. 1, 2008, pp. 217-221. doi:10.1007/s00339-008-4485-3

[29] B. Hopp, Z. S. Bor, E. Homolya and E. Mihalik, "Investigation of Conical Structures Created by ArF Excimer Laser Irradiation of Polycarbonate," Applied Surface Science, Vol. 109-110, No. 1, 1997, pp. 232-235. 


$$
\text { doi:10.1016/S0169-4332(96)00915-4 }
$$

[30] Y. G. Yingling and B. J. Garrison, "Coarse-Grained Model of the Interaction of Light with Polymeric Material: Onset of Ablation," Journal of Physics Chemistry B, Vol. 109, No. 34, 2005, pp. 16482-16489. doi:10.1021/jp0527711

[31] R. Srinivasan, M. A. Smrtic and S. V. Babu, "Excimer Laser Etching of Polymers," Journal of Applied Physics, Vol. 59, No. 11, 2005, pp. 3861-3867. doi: $10.1063 / 1.336728$

[32] B. Lukyanchuk, N. Bityurin, S. Anisimov, N. Arnold and D. Bäerle, "The Role of Excited Species in UltravioletLaser Materials Ablation III. Non-Stationary Ablation of Organic Polymers," Applied Physics A, Vol. 62, No. 5, 1996, pp. 397-401. doi:10.1007/BF01567110

[33] D. J. Krajnovich, "Near-Threshold Photoablation Characteristics of Polyimide and Poly(Ethylene Terephtha- late)," Journal of Applied Physics, Vol. 82, No. 1, 1997, pp. 427-435. doi:10.1063/1.366290

[34] J. D. Spear and R. E. Russo, "Transverse Photothermal Beam Deflection within a Solid," Journal of Applied Physics, Vol. 70, No. 2, 199, pp. 580-586. doi: $10.1063 / 1.349659$

[35] S. W. Wee and S. M. Park, "Laser Ablation of Poly(Methyl Methacrylate) at $266 \mathrm{~nm}$," Bulletin Korean Chemistry Society, Vol. 22, No. 8, 2001, pp. 914-916.

[36] E. Skantzakis, V. Zorba, D. G. Papazoglou, I. Zergioti and C. Fotakis, "Ultraviolet Laser Micro-Structuring of Silicon and the Effect of Laser Pulse Duration on the Surface Morphology," Applied Surface Science, Vol. 252, No. 13, 2006, pp. 4462-4466. doi:10.1016/j.apsusc.2005.07.120 